\title{
The significant influence of surface states on the electroluminescence of CdS nanoparticles
}

\author{
Eih-Zhe Liang ${ }^{\mathrm{a}}$, Ching-Fuh Lin*a, Sheng-Ming Shih ${ }^{\mathrm{b}}$, Wei-Fang $\mathrm{Su}^{\mathrm{b}}$ \\ ${ }^{a}$ Graduate Institute of Electro-Optical Engineering \\ ${ }^{\mathrm{b}}$ Institute of Materials Science and Engineering \\ National Taiwan University, Taipei 106, Taiwan, ROC
}

\begin{abstract}
The significance of surface states in nano-structures is studied using CdS nanoparticles. Spectral features like peak red-shift due to organic capping and influence of surface states have been observed. The pronounced enhancement of emission from surface states can be dominant with certain modification of CdS nanoparticles. Spectral behaviors of electroluminescence in different temperature are also studied.
\end{abstract}

Keywords: CdS nanoparticles, low-dimensional structure, surface states, electroluminescence, exciton.

\section{INTRODUCTION}

Nanoparticles formed by chemical methods have certain advantages. They have low cost of production and well control physical properties. Compared with epitaxial quantum dots, nanoparticles can be simply dissolved in various solutions and applied to nonspecific substrates. Stimulated emission and optical gain had been reported with CdS quantum dots by optical pumping ${ }^{1,2}$. However, it is challenging to employ electrical pumping to realize nanoparticlebased light emitting devices. Electroluminescence from CdS nanoparticles reported in this work proves the feasibility of using such materials as active light-emitting media.

Since nanoparticles are low-dimensional materials $(\sim 5 \mathrm{~nm})$, compared with bulk materials, it results in large surface contact area with environment. Surface states formed by termination with oxygen or other contaminants are thus of great amount. Generally this situation is avoided by using passivation around nanoparticles, in our case organic p-hydroxyl thiophenol group. However, we found that there can be significant enhancement of light emission from surface states. It can be useful in addition to intrinsic quantum states provided by low-dimensional structures.

Electroluminescence of CdS nanoparticles in different environment such as normal treatment, heat treatment and oxygen enrichment is achieved. Emission spectrum of CdS nanoparticles is significantly influenced by both process temperature and oxygen surrounding condition. Radiative recombination due to free exciton in CdS is observed, with spectral peak shift due to organic encapping. As surrounding oxygen content level is raised, radiative recombination from surface states emerges. Side effect such as coalescence of CdS nanoparticle into bulk form also presents in raising process temperature. At different temperature, the EL spectrum of CdS nanoparticle remains quite the same. Peak shift is compared with bulk bandgap shift and ascribed as effect of quantum confinement and surface configuration.

This work also demonstrates the electroluminescence of CdS-nanoparticle on silicon substrate. The fabrication of light emitting active layer is simply the spin-coating technique. Carriers to achieve light emission can be supplied by quantum tunneling through surrounding barrier into nanoparticles. Using silicon as substrate shows a promising way to monolithically integrate light emission of nanoparticles and conventional electrical circuitry. Moreover, optical functional blocks can be built at relatively low temperature, following traditional process. This advantage eliminates conflicted thermal budget of epitaxial III-V materials and silicon circuitry.

\section{SIGNIFICANCE OF SURFACE STATES}

In the unpassivated case, nanoparticle interacts with oxygen as exposed to atmosphere. Surface states associated with oxygen, in our case $\mathrm{Cd}-\mathrm{O}$ bond, are formed. The ratio between surface area and volume ratio of nanoparticle increases as individual particle size shrinks into several nanometer range. Radiative recombination rate with surface

* Email:cflin@cc.ee.ntu.edu.tw; also with Graduate Institute of Electronics Engineering and Department of Electrical Engineering 
states origin becomes comparable with that of CdS core, or even dominant. To be specific, assuming $\mathrm{n}$ is the number of atoms along the radius of nanoparticle, the ratio of atoms in the top surface layer to core atoms is given by

$$
\left(n^{3}-(n-m)^{3}\right) /(n-m)^{3}
$$

where $m$ is effective number of atoms of surrounding surface layer. We can see in Fig. 1 that the ratio becomes unity as the radius shrinks within $n \leq 10$. In such cases, radiative recombination rate of surface states becomes comparable to intrinsic ones, assuming alike oscillation strength. This situation describes how the unpassivated CdS nanoparticles can emit more light with surface state origin than that observed in epitaxial or bulk CdS case.

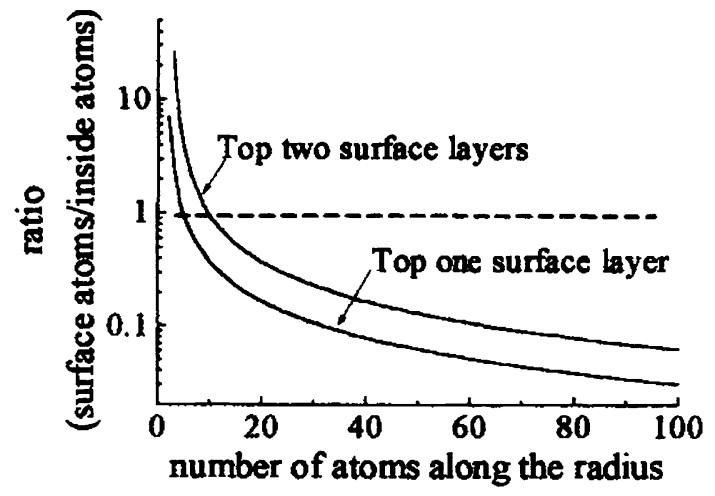

Fig. 1 Ratio of surface atoms to core atoms with respect to number of atoms along radius.

\section{PREPARATION OF MATERIALS}

Two kinds of CdS nanoparticles ready for spin-coating purpose are synthesized by modification of Pietro's method ${ }^{3}$. First form is CdS nanoparticle with organic capping. Cadmium acetate dihydrate $\mathrm{Cd}\left(\mathrm{CH}_{3} \mathrm{COO}\right)_{2} 2 \mathrm{H}_{2} \mathrm{O}, 0.80 \mathrm{~g}$, $3.0 \mathrm{mmole}$ ) is dissolved in $20 \mathrm{ml}$ mixed solvent of acetonitrile, methanol, and water with volume ratio 1:1:2. Another solution containing disodium sulfide nanohydrate $\left(\mathrm{Na}_{2} \mathrm{~S} 9 \mathrm{H}_{2} \mathrm{O}, 0.36 \mathrm{~g}, 1.5 \mathrm{mmole}\right)$ and p-hydroxyl thiophenol $(0.56 \mathrm{~g}$, $4.4 \mathrm{mmole}$ ) in the same solvent system is added into vigorously stirred cadmium acetate solution. The whole system was stirred for 18 hours without light illumination. After removing solvent and purifying by centrifuge, we obtained $0.70 \mathrm{~g}$ yellow solid aggregate of CdS nanoparticles capped by p-hydroxyl thiophenol.

Second form of CdS nanoparticle is coated with silica shell. The purpose of preventive use of organic component is to raise the thermal budget of whole fabrication process and increase tolerance with low temperature. The preparation process is as follows. Cadmium acetate dihydrate $\left(\mathrm{Cd}_{\left(\mathrm{CH}_{3} \mathrm{COO}\right)}{ }_{2} 2 \mathrm{H}_{2} \mathrm{O}, 1.60 \mathrm{~g}, 6.0 \mathrm{mmole}\right)$ is dissolved in $32 \mathrm{ml}$ mixed solvent of acetonitrile and water with volume ratio 1:1. Another solution containing disodium sulfide $\left(\mathrm{Na}_{2} \mathrm{~S} \mathrm{xH}_{2} \mathrm{O}\right.$, $\mathrm{x}=7 \sim 9,0.58 \mathrm{~g}, \sim 2.4 \mathrm{mmole})$ and ( $\gamma$-mercaptopropyl) trimethoxysilane $(1.41 \mathrm{~g}, 7.2 \mathrm{mmole})$ in the same solvent system is added into vigorously stirred solution of cadmium acetate. After being vigorously stirred for 18 hours, the mixture is basified to $\mathrm{pH}=8.4$ with $25 \%$ of $\mathrm{NH}_{3}$ aqueous solution. Additional $64 \mathrm{ml}$ of ethanol is added to the mixture. The mixture is stirred for 48 hours after adding $1.89 \mathrm{~g}$ of orthotetraethoxysilane (TEOS). Part of the solvent was removed and precipitation takes place in the mixture. The precipitate is centrifuged for three times and rinsed with deionized water.

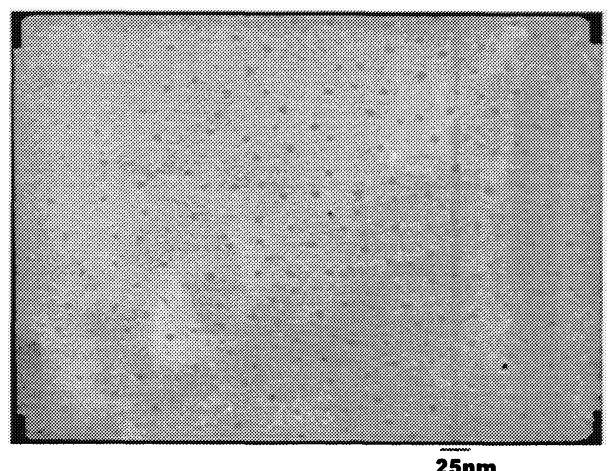

Fig. 2: TEM image of p-hydroxyl thiophenol capped CdS nanoparticles. 
The prepared nanoparticle aggregate is redispersable in ethanl or other polar organic solvents. After treated by ultrasonic vibration and percolation, solutions for spin-coating purpose are produced.

To take TEM image, prepared solution is dipped onto carbon film coated copper plate and reabsorbed. Sparsely distributed nanoparticles are obtained. The average diameter of the spherical CdS nanoparticles is about $5 \mathrm{~nm}$, as shown in Fig. 2. Compared with the high-temperature synthesizing method by using trioctylphosphine oxygen (TOPO), this room-temperature process is easier but the particle size distribution is wider.

By replacing part of cadmium acetate with manganese acetate, we prepared Mn doped CdS nano- particles with different concentrations of manganese $(5 \%, 10 \%$ and $20 \%$, in molar percentage). Through out the experiment, no significant difference of doped and undoped CdS nanoparticles is found under the spectral resolution of our monochromator. To synthesize manganese doped CdS nanoparticles, we have used large molar percentage of $\mathrm{Mn}, 5 \%$, $10 \%$ and $20 \%$ respectively in the reaction. However, only trace doping amounts of $\mathrm{Mn}, 0.08 \%, 0.05 \%$ and $1.10 \%$ was detected respectively by ICP-Mass investigations. When the same approach was used to synthesize MnS nanoparticles, a very low yield was obtained. This indicates very low doping content can be made and electroluminescence is less affected by Mn content.

\section{SETUP FOR ELECTROLUMINESCENCE}

The basic idea about setup of electroluminescence is to use cascade tunneling as injection source. Carriers are supplied by tunneling current through potential barrier of high bandgap material surrounding nanoparticle, as shown in Fig. 3. In our case, the $\mathrm{SiO}$ group or organic functional group p-hydroxyl thiophenol serves as the goal. The choice of substrate to host CdS nanoparticles is silicon due to its surface quality and availability. In addition, in this early study of light emission, difficulty to maintain uniformity of film exists if nanoparticle solution of aqueous solvent system is used. The Schottky barrier of metal-silicon contact has certain prevention from short circuit other than indium-tin-oxide (ITO) glass.

With different type of injection carriers, depending on n-type or p-type silicon substrate, electrons or holes are emitted from silicon and holes or electrons are emitted from metal deposited. Fermi level of Si in n-type case has to be raised for electrons to tunnel through potential barrier of p-hydroxyl thiophenol group. Most carriers tunnel into the adjacent nanoparticles. Carriers are expected to recombine within nanoparticles through intrinsic radiative transition or surface-state related transition.

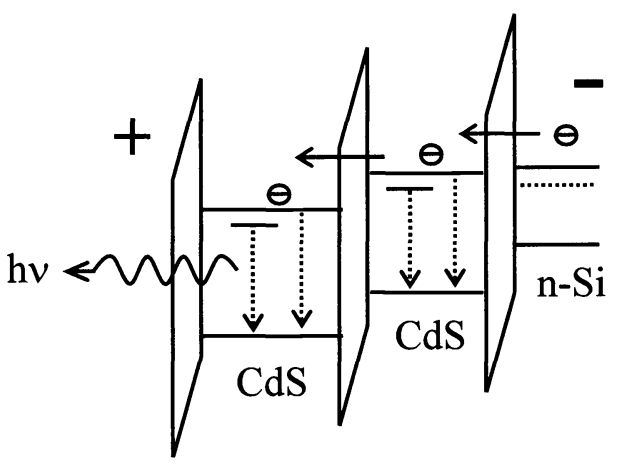

Fig. 3: Schematic of electron transport and transition in the device.

A schematic of CdS-nanoparticle light emitting stage is shown in Fig. 4. The fabrication steps are as follows. First, a doped silicon wafer (doping $\sim 10^{15} \mathrm{~cm}^{-3}$ ) is used as substrate. Acetone, methanol, and DI water are used successively for clean procedure. Buffered oxide etch is applied to remove native oxides. The wafer is spin-coated with CdS nanoparticle solutions. Solvents are either removed by evacuation or heat treatment. The thickness of CdS nanoparticle layer can be as large as $500 \mathrm{~nm}$, verified by surface profile scan. In this case, volume density can be very high.

Subsequently, both top and bottom metal contacts are made by thermal evaporation. The top semi-transparent contact layer is $10 \mathrm{~nm}$ gold, and the bottom is $150 \mathrm{~nm}$ gold. Before the deposition of the Au layer, a 3-nm adhesion layer of chromium had been evaporated for both contacts. A CM110 monochromator and photomultiplier is used to record the electroluminescence spectrum. In every spectrum measurement, the entrance slit width $0.6 \mathrm{~mm}$ is used for maximum detection and correct spectrum. 


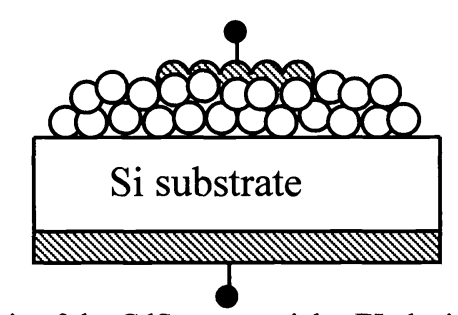

Fig. 4: Schematic of the CdS nanoparticles EL device on Si wafer.

Samples made on $\mathrm{n}$ - and p-type Si wafers show different current-voltage curves, as shown in Fig. 5. Both have rectifying current-voltage (I-V) curves, but with opposite polarities. This rectifying effect corresponds to metal-insulator-semiconductor tunneling effect as expected. To be specific, the thin potential barrier of organic functional group and low substrate doping level results in Schottky-diode-like behavior.

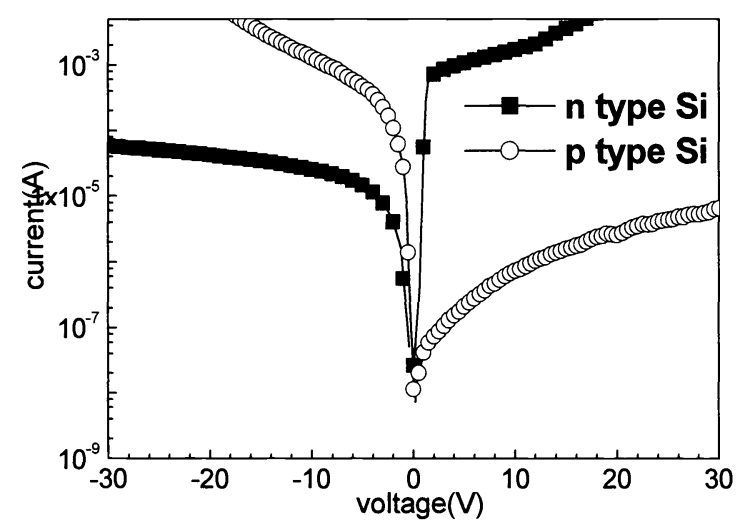

Fig. 5: I-V curves of devices on n-type and p-type Si.

\section{PROPERTIES OF ELECTROLUMINESCENCE}

We take different post treatments after the nanoparticle film is spin-coated onto silicon substrate. The normal treatment is to remove the solvent without physical change of nanoparticles. Heat treatment is carried out in medium high temperature to explore decomposability of organic passivation and test oxygen cooperation. Oxygen enrichment raises extent of oxygen related surface states. All three conditions show prominent spectral features and can be used to monitor passivation of surface condition.

Origins of electroluminescence of CdS nanoparticle with bulk CdS are schematically shown in Fig. 6. Three exciton levels corresponding to bulk CdS bandgap now change their peak positions of quantum states due to modification of surface configuration. Modified A free exciton level changes from $508 \mathrm{~nm}$ to $526.5 \mathrm{~nm}$ as can be determined in normal treatment of electroluminescence device. Transition level of surface states related to $\mathrm{Cd}-\mathrm{O}$ termination with peak position of $571.5 \mathrm{~nm}$ can be observed. This level occurs when passivation of nanoparticle is removed and it has contact with atmosphere, as in heat treatment and oxygen enrichment.

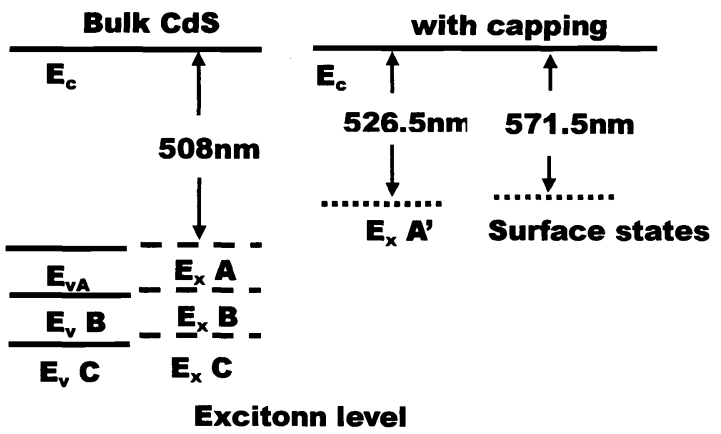

Fig. 6 Energy diagram of CdS nanoparticle. 


\subsection{Normal process}

After spin-coated, the device is placed in a chamber with evacuation at room temperature for 5 minutes to remove ethanol solvents. Both spectra of CdS and CdS doped with Mn are the same, as shown in Fig. 7. The spectrum fits into Lorentzian shape with scattering time of 6 fs. Its FWHM is $42 \mathrm{~nm}$. Such broad spectrum indicates the dispersion of particle size and a trade-off of low temperature synthesis.

This spectral peak indicates radiative recombination of free exciton in CdS nanoparticles with red-shift due to p-hydroxyl thiophenol groups. The green spectral peak is at $526.5 \mathrm{~nm}(2.355 \mathrm{eV})$, different from bulk CdS A-exciton transition energy, $2.441 \mathrm{eV}(508 \mathrm{~nm})$ at room temperature. Although quantum confinement within the nanoparticles increases exciton energy whenever the particle size decreases ${ }^{4,5}$, organic functional group or silicon dioxide matrix can modify the electron configuration within and change ground states significantly. It results in energy red-shift of $86 \mathrm{meV}$. CdS nanoparticles coated with poly(vinyl alcohol) also show such energy shift in absorption spectrum ${ }^{6}$, where photoluminescence at $2.42 \mathrm{eV}(10 \mathrm{~K})$ is observed.

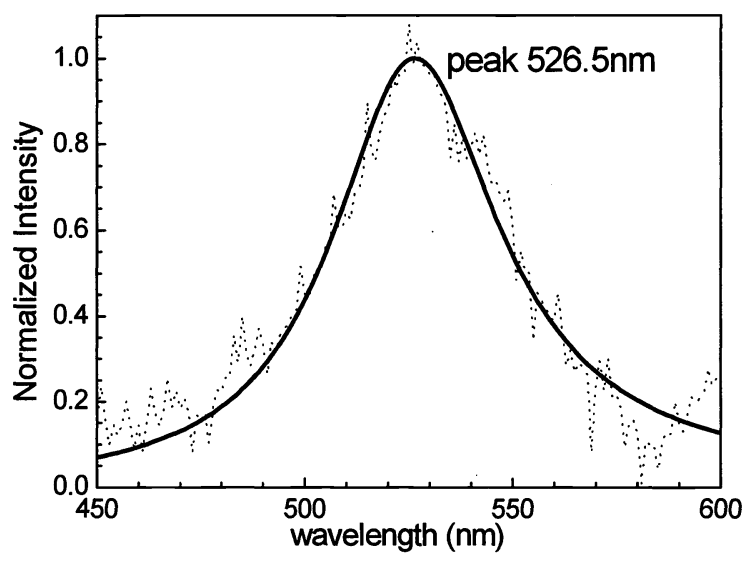

Fig. 7: EL Spectrum of CdS with solvent removed.

\subsection{Heat treatment}

The CdS nanoparticles are spin-coated as described in previous section. These samples are subsequently treated by rapid thermal annealing (RTA) with temperature $425^{\circ} \mathrm{C}$ for 5 minutes. The annealing process takes place in nitrogen purge and its purpose is to remove solvent and both test decomposition of organic functional group. Electrical property like I-V curve resembles that in Sec. 3.1. As shown in Fig. 8 the emission spectrum consists of two peaks. One is at $513.7 \mathrm{~nm}$ and the other at $571.5 \mathrm{~nm}$. The former peak stands for bulk CdS free A exciton transition. This spectral lobe can be fitted by Lorentzian shape with scattering time of $8 \mathrm{fs}$ and FWHM $40 \mathrm{~nm}$.

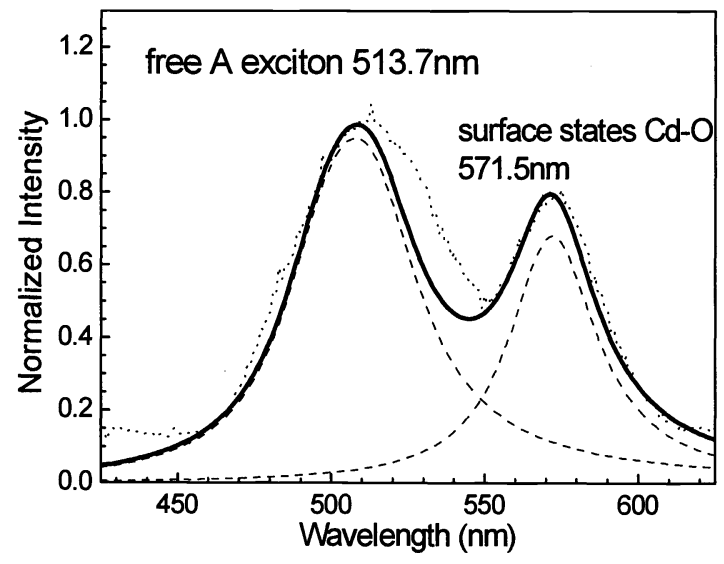

Fig. 8: The EL Spectrum of CdS particles after heat treatment. 
The $571.5 \mathrm{~nm}$ peak results from the trapped carriers in surface states related to oxygen, Cd-O termination ${ }^{7}$. In medium high temperature treatment, decomposition of p-hydroxyl thiophenol group causes oxygen termination with cadmium to occur. It proves p-hydroxyl thiophenol group to be effective overcoat of CdS nanoparticles against oxygen influence. The surface states induce radiative transition as well. The peak magnitude at the spectral lobe is smaller than the magnitude at $513.7 \mathrm{~nm}$, indicating emission from surface states is weaker than that resulted from CdS nanoparticles. However, light power from this sample is generally stronger than that in Sec. 3.1. This phenomenon is due to the participation of surface level luminescence, leading to increase of total light output.

\subsection{Effect of surrounding oxides}

To further study the surface states related to oxygen, we immersed CdS nanoparticles into high oxygen content environment. Two ways are proceeded. First, the nanoparticle solutions were mixed with spin-on-glass (SOG, Filmtronics $315 \mathrm{FX}$ ), and the second way, mixed with $\mathrm{SiO}_{2}$ nanoparticles (average diameter of $12 \mathrm{~nm}$, dissolved in isopropanol). The cleaned, oxide-free silicon substrate is spin-coated and treated by $425^{\circ} \mathrm{C}$ to sinter with $\mathrm{SiO}_{2}$ glass. The similar EL spectrum is found in mixture of CdS nanoparticles with $\mathrm{SOG}$ and $\mathrm{SiO}_{2}$ nanoparticles. The peak at $513.7 \mathrm{~nm}$ $(2.414 \mathrm{eV})$ resembles $\mathrm{A}$ free exciton signal of bulk $\mathrm{CdS}$ at temperature $65^{\circ} \mathrm{C}$, and the peak at $571.5 \mathrm{~nm}(2.414 \mathrm{eV})$ corresponds to radiative transition due to surface states. The magnitude of total light emission in current setups is ten times stronger than that of unheated samples in Section 3.1, under the same carrier injection condition.

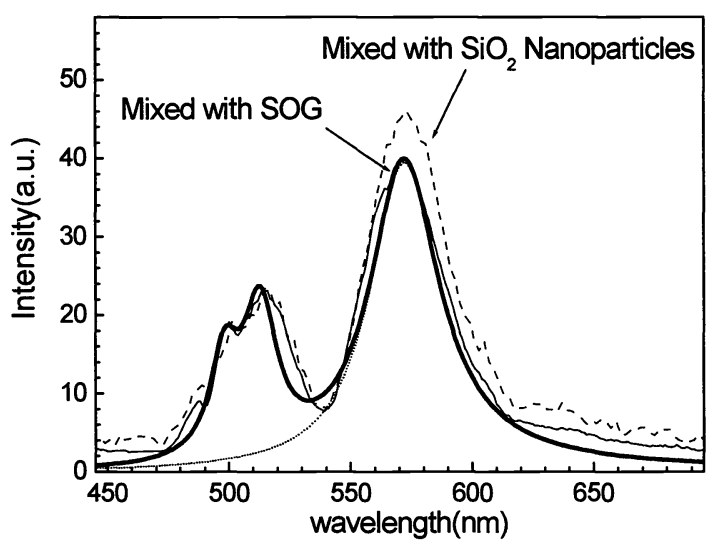

Fig. 9: EL spectrum of CdS nanoparticles with oxygen enrichment

EL spectrum shown in Fig.9 indicates two mechanisms as shown by the energy diagram in Fig. 6. First, the coalescence of CdS nanoparticles into bulk form results in less broadening spectrum around $513.7 \mathrm{~nm}$. Since the potential barrier of p-hydroxyl thiophenol group disappears due to decomposition, carriers in bulk powders stay for enough time (about 1 ns transition lifetime) to recombine radiatively between each tunneling process. Second, relative magnitude of surface states luminescence is much stronger than that in Sec. 3.2. Highly increased concentration of surface state levels, which are supplied by surrounding oxygen termination, contributes to the enhancement of internal quantum efficiency. The magnitude difference between mixture with $\mathrm{SOG}$ and $\mathrm{SiO}_{2}$ nanoparticles comes from excess dangling Si-O bond of the latter case. With the same sintering time, the latter mixture makes more extent of oxygen enrichment.

\subsection{Temperature effect}

To examine EL property of CdS nanoparticle with varied temperature, original organic functional group is substituted with inorganic silica passivation shell, in order to prevent instability of organic composition at low temperature. EL spectra of silica-passivated CdS nanoparticles are shown in Fig. 10. The resembling peak of surface states represents $\mathrm{Cd}-\mathrm{O}$ termination, now introduced by silica passivation at surrounding surface probes to origin of light emission.

As temperature increases, the EL intensity decreases. Such reduced emission efficiency comes from increased nonradiative mechanism with increased temperature. Surface states and carrier-phonon scattering both play roles. Ten times stronger magnitude of light emission intensity at low temperature of $15 \mathrm{~K}$ compared with room temperature case reveals that nonradiative mechanism is a crucial factor influencing emission efficiency of the emitter. The reason for the 
similar spectra is due to two causes. First, the shift of quantized energy levels due to small nanoparticles $(<5 \mathrm{~nm})$ contributes to the spectrum around $520 \mathrm{~nm}$. Second, transition of surface states may have multiple levels corresponding to broad spectrum at room temperature.

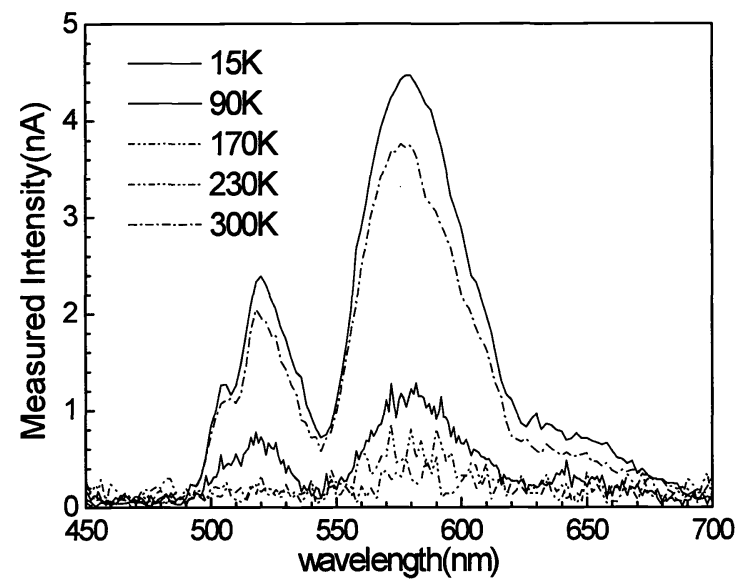

Fig. 10: EL spectrum of CdS nanoparticles with silica shell at varied temperature.

Spectral peak at $520 \mathrm{~nm}$ in Fig. 10 is attributed to the same origin as peak at $526.5 \mathrm{~nm}$ in organic-capped CdS nanoparticles, with additional effect of quantum confinement and surface configuration. As a result, peak shift with varied temperature is compared with bulk bandgap shift in Fig. 11 and ascribed as characteristics of as-synthesized CdS nanoparticle. Ten times increase of emission intensity is observed at cryogenic temperature (15K), indicating strong influence of surface traps due to the large surface area. Reduced thermal scattering also contributes to enhancement of light emission at low temperature. The relative magnitude of recombination rate of surface states to exciton level changes slightly and may result from its nearness to conduction band

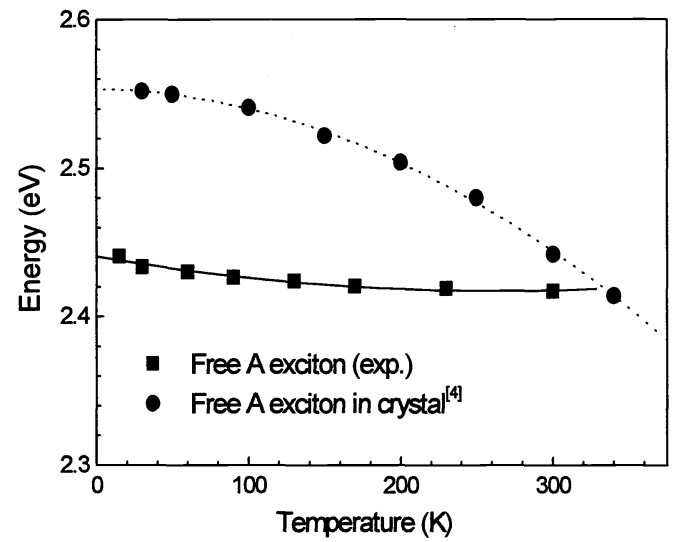

Fig. 11: Spectral peak variation with temperature compared with bulk exciton energy.

With energy parameters of bulk CdS given above ${ }^{6}$, red-shift due to p-hydroxyl thiophenol group is determined as $86 \mathrm{meV}$. Also, transition level of surface states is found to be $273 \mathrm{meV}$ below bulk bandgap. In addition to observation of such peak at $571.5 \mathrm{~nm}$, similar phenomena at spectral range, $550 \mathrm{~nm} \sim 600 \mathrm{~nm}$, as an indication of imperfect CdS crystal or nanoparticles had been reported elsewhere. Okamoto ${ }^{8}$ also ascribed their broad peak at $650 \mathrm{~nm}(1.9 \mathrm{eV})$ to surface state emission. Hong ${ }^{7}$ also demonstrated broad peak of $592 \mathrm{~nm}(2.0944 \mathrm{eV})$ due to S-vacancy (in this case termination of oxygen).

\subsection{Raman spectrum}

Previously observed transition of manganese ion in CdS nanoparticle at $585 \mathrm{~nm}(2.119 \mathrm{eV})^{9}$ is not clearly observed in our samples, mainly due to insufficient spectral resolution and trace content of Mn incorporated to CdS core. To 
determine the factor on firm ground, Raman spectroscopy is taken as shown in Fig. 12.

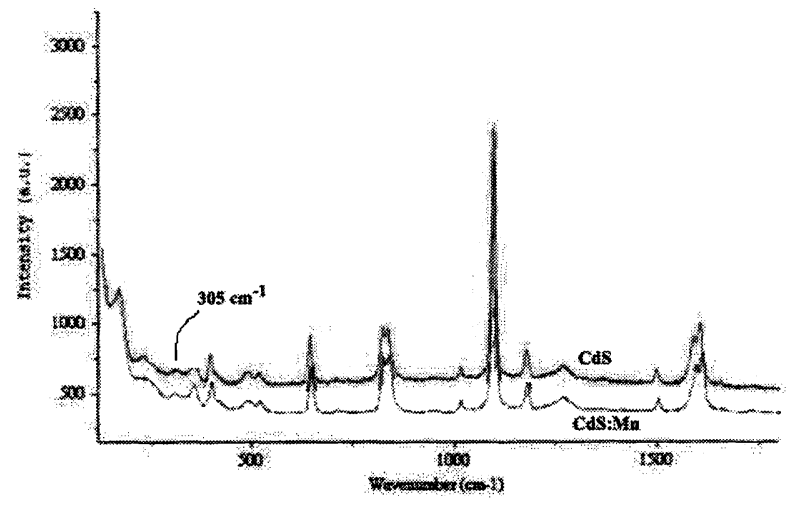

Fig. 12 Raman spectrum of CdS nanoparticles as synthesized.

Raman shift at $305 \mathrm{~cm}^{-1}$ corresponds to the longitudinal optical phonons LO) mode of bulk $\mathrm{CdS}^{10}$. No wave number shift is observed for CdS/Mn nanoparticles, therefore $\mathrm{Mn}$ is not in the CdS core lattice. The other Raman shift peaks are from the vibrations of the organic molecules. Consequently, the EL peaks of CdS nanoparticles prepared with or without addition of $\mathrm{Mn}$ are the same as shown in Figure 7.

We have mentioned reaction rate of MnS synthesis is slower than that of CdS synthesis. Therefore, the position of $\mathrm{Cd}$ in the lattice of CdS can barely be replaced by Mn. It is trapped by the hydroxyl group of p-hydroxyl thiophenol capping on the surface of CdS nanoparticles.

\section{CONCLUSIONS}

Significant surface influence is observed for nanoparticles. Chemical preparation of CdS nanoparticles ready for spin-coating and LEDs made of CdS and CdS:Mn nanoparticles on Si substrates are described in detail. EL properties are investigated. Spectral shift of free exciton transition due to passivation of p-hydroxyl thiophenol group around nanoparticles is discovered. Process modifications such as heat treatment and oxygen enrichment are influential to intrinsic green emission. P-hydroxyl thiophenol group is shown to have protection from diffusion of contaminants into nanoparticles, but cannot resist temperature deterioration above $400^{\circ} \mathrm{C}$.

Radiative recombination of carriers trapped in surface states present and magnifies itself as long as extent of surface states increases. Ten times increase of emission intensity is observed at cryogenic temperature (15K), indicating strong influence of surface traps due to the large surface area. Reduced thermal scattering also contributes to enhancement of light emission at low temperature. At varied temperature, the EL spectrum of CdS nanoparticle remains quite the same. Peak shift is compared with bulk bandgap shift and ascribed as effect of quantum confinement and surface configuration.

\section{ACKNOWLEDGEMENTS}

The authors acknowledge the support from National Science Council, ROC under the contract number NSC 90-2215-E-002 and NSC 90-2622-L002.

\section{REFERENCES}

1. V.I. Klimov, A.A. Milkhailovsky, Su Xu, A. Malko, J.A. Hollingsworth, C.A. Leatherdale, H.-J. Eisler, and M.G. Bawendi, "Optical Gain and Stimulated Emission in Nanocrystal Quantum Dots ," Science 290, pp.314-317, 2000.

2. J. Butty, Y.Z. Hu, N. Peyghambarian, Y.H. Kao, and J.D.Mackenzie, "Quasicontinuous gain in sol-gel derived CdS quantum dots ," Appl. Phys. Lett. 67, pp.2672-2674, 1995.

3. J. G. C. Veinot, M. Ginzburg, and W. J. Pietro, "Surface Functionalization of Cadmium Sulfide Quantum-Confined Nanoclusters. 3. Formation and Derivatives of a Surface Phenolic Quantum Dot,"Chem. Mater. 9, pp.2117-2122, 1997.

4. B.G. Potter, Jr. and J.H. Simmons, "Quantum size effects in optical properties of CdS-glass composites,” Phys. Rev. B 
37, pp.10838-10845, 1988.

5. M.V Rama Krishna, and R.A. Friesner, "Quantum confinement effects in semiconductor clusters," J. Chem. Phys. 95, pp.8309-8322, 1991.

6. M. Tanaka, J. Qi, and Y. Masumoto, "Optical properties of undoped and $\mathrm{Mn}^{2+}$-doped CdS nanocrystals in polymer," $J$. Crystal Growth 214/215, pp.410-414, 2000.

7. K.J. Hong, T.S. Jeong, C.J. Yoon, and Y.J. Shin, "The optical properties of CdS crystal grown by the sublimation method," J. Crystal. Growth. 218, pp.19-26, 2000.

8. S. Okamoto, Y. Kanemitsu, H. Hosokawa, K. Murakoshi, and S. Yanagida, "Photoluminescence from suface-capped CdS nanocrystals by selective excitation ," Solid State Commun. 105, pp.7-11, 1998.

9. L. Spanhel, E. Arpac, and H. Schmidt, "Semiconductor clusters in the sol-gel process. Synthesis and properties of CdS nanocomposites," J. Non. Cryst. Solids 147/148, pp.657-662, 1992.

10.K.K. Nanda, S.N. Sarangi, S.N. Sahu, S.K. Deb and S.N. Behera, "Raman spectroscopy of CdS nanocrystalline semiconductors," Physica B 262, pp.31-39, 1999. 